RePortes de Tesis De Posgrado

\title{
Modelado tridimensional de vasijas polícromas y urnas mixtecas
}

\author{
Noé Coronel García
}

Publicado: 21 Septiembre 2016

\begin{abstract}
Resumen
La presente investigación pretende mostrar el trabajo que puede realizarse para difusión y preservación de la cultura, utilizando la tecnología a nuestro alcance. Es una mezcla de conocimientos en Interacción Humano Computadora, Experiencias de Usuario, Arqueología, Fotografía y diseño en 3D. La presentación del trabajo de modelado se realizará de acuerdo a las experiencias que se obtengan del grupo de usuarios, clasificados en: arqueólogos, visitantes a los museos y guías.
\end{abstract}

\section{Palabras clave: Vasijas;3D; UCD; INAH.}

\section{Introducción}

La Mixteca, una región que por años estuvo aislada cultural y geográficamente, abarca tres Estados de la República Mexicana (Oaxaca, Guerrero y Puebla) y no cuenta con un gran acervo digital que vincule su historia de manera virtual en entornos 3D. Así como tampoco estudios etnográficos que aborden el desarrollo y las particularidades de los grupos etnolingüísticos, que puedan utilizarse en la divulgación del conocimiento apoyados en el uso de tecnologías actuales, como el modelado 3D y la realidad aumentada.

Para ello, es necesario recuperar, estructurar y analizar la información histórica, geográfica y arqueológica, obtenida hasta el momento por personal del Instituto Nacional de Antropología e Historia (INAH).

Haciendo énfasis en la Cultura Mixteca, principalmente la asentada en Oaxaca, una de las regiones mesoamericanas de mayor profundidad histórica. A lo largo de aproximadamente 3,000 años, en ese territorio se desarrollaron dos de las más grandes tradiciones culturales, la Mixteca y la Zapoteca, las cuales se encuentran entre las más importantes de Mesoamérica por su longevidad y el alcance de sus aportaciones [4]. Hoy en día, esa extraordinaria diversidad cultural se refleja en los numerosos grupos étnicos que aún pueblan la entidad, en lo que de hecho constituye el conglomerado indígena más numeroso del país, con una notable variedad de lenguas, así como de costumbres y creencias con raíces prehispánicas [5].

\footnotetext{
Coronel García, N.

Universidad Tecnológica de la Mixteca

Carretera a Acatlima, km. 2.5

Huajuapan de León, Oaxaca, México.

Email: noegarcia2@gmail.com
}

\section{Trabajo relacionado}

Desde el año 2009 el INAH inició con un proyecto de modelado tridimensional denominado "pon a México en el mapa". En dicha convocatoria se presentan modelados elaborados en tres dimensiones que corresponden a temas diversos. Los materiales son resultado de proyectos de investigación y de la convocatoria hecha a estudiantes universitarios, para modelar estructuras prehispánicas y monumentos históricos bajo resguardo del Instituto [7].La mayoría de los modelados corresponden a catedrales, templos, pirámides, zonas arqueológicas, y se debe de tener un alto grado de realismo para alcanzar los primeros lugares.

En trabajos de reconstrucción o modelados virtuales, la Cultura Maya es de las más consultadas para dichos trabajos, los cuales se han realizado en ciudades de arquitectura e influencia Maya. En este caso, la Cultura Mixteca ha estado un poco alejada de dichas investigaciones y trabajos, a pesar de que existe una gran cantidad de información que ha sido obtenida por los arqueólogos del INAH y los diferentes museos que se encuentran en dicha región, los cuales forman parte del objetivo del presente trabajo.

\section{Objetivo de la investigación}

El objetivo general del presente trabajo de tesis se puede resumir como: "Crear un modelado tridimensional de vasijas polícromas y urnas mixtecas que permita comprender la importancia de las artesanías prehispánicas de la Cultura Mixteca, ubicadas en nuestra región."

Al realizar el presente proyecto se pretende modelar en tres dimensiones específicamente urnas y vasijas polícromas, que son las que le dieron el reconocimiento de artesanos a los mixtecos que conquistaron con el arte los lugares que no pudieron con la guerra. Para tal fin, es necesario recuperar, analizar, estructurar y modelar la información obtenida por personal del INAH. Las piezas seleccionadas para el modelado serán evaluadas por criterio de los arqueólogos y se tomaran en cuenta factores como: grado de importancia, el gusto entre los usuarios, y el estado de conservación de la policromía en la pieza.

La Figura 1, muestra una vasija polícroma, perteneciente al grupo de ollas trípodes, donde predominan los colores claros, diseñando nubes, flores y cabezas de serpientes, todas formadas con volutas. La pieza muestra serpientes de perfil que van a repetirse muy a menudo en las vasijas mixtecas. En la misma pieza pueden observarse diseños de nubes en el cuello. Se considera posible que este diseño de nubes sea emblema de la nación y pueblos mixtecos, que se llaman a sí mismos "gente de 
las nubes". Dada la importancia de esta Cultura y lo relevante que es para la preservación de la misma, es lo que motiva la realización de este proyecto, con el fin de generar material tridimensional que permita difundirla y preservarla a través de la educación.

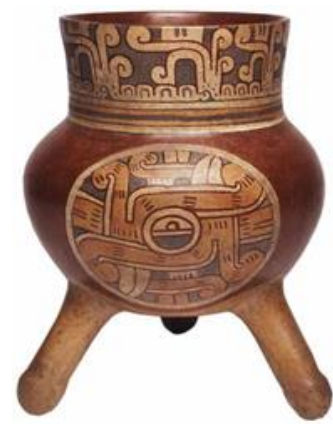

Figura 1. Vasija trípode polícroma. Después de 950 d.C., en la Mixteca ocurrió una revolución cultural y artística que permitió a los mixtecos crear la delicada cerámica polícroma. MNA. Foto: Marco Antonio Pacheco / Raíces

El desarrollo de una aplicación, que deba ser aplicada o utilizada en un ambiente virtual, requiere de un modelo de datos apropiado para manejar la información del mundo real, flexible, extensible y con la capacidad para representar información del mundo real y virtual [1]. Modelos más apropiados impactarán positivamente en el tiempo de desarrollo, resultando más efectivos, prestando un mayor beneficio al usuario, y es parte de lo que se pretende.

\section{Metodología}

El Diseño Centrado en el Usuario (DCU) o UCD del inglés (UserCenteredDesign) ha cobrado popularidad en los últimos años como proceso encaminado al diseño de productos, objetos o servicios, que respondan a las necesidades reales de sus usuarios finales [2].

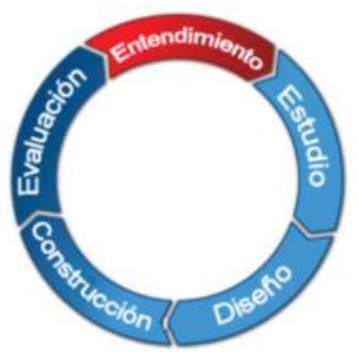

Figura 2. Fases de la Metodología UCDe [6]

La Figura 2, muestra lo que proponen en [6], mediante la adición de una nueva etapa, que implica el análisis conceptual. En esta nueva etapa se necesita saber más acerca de las experiencias de la gente, los usuarios finales, es aquí donde los estudios etnográficos, registros de interacción con el usuario y las encuestas se usan comúnmente. Es en esta metodología estará basada la investigación que dará soporte a nuestro modelado.

Para la realización del presente proyecto, se cuenta con el apoyo de personal del INAH, gestores ubicados en Huajuapan de León, arqueólogos de la ciudad de Oaxaca, Museo de las Culturas de Oaxaca y un arqueólogo con sede en la ciudad de México, quienes proporcionarán datos que tienen de las excavaciones y los estudios que se han venido realizando en diferentes sitios de la mixteca, con vasijas policromas y urnas mixtecas.

\section{Resultados actuales}

- Fase 1: Acopio de información y análisis de datos. Los objetos físicos de nuestro estudio, conservan un estado diferente al que tuvieron cuando fueron hechas, por lo cual al realizar el análisis de manera conjunta con los arqueólogos se ha tratado de reproducir de la manera más fiel el modelo físico que permita apreciar el modelado en 3D. En esta fase ha sido fundamental el entendimiento entre el desarrollador y los arqueólogos.

- Fase 2: Aplicación y desarrollo de la metodología UCDe. El presente proyecto de modelado tridimensional busca destacar las características físicas de las obras artesanales de dicha cultura. Por lo cual basados en UCDe se ha obtenido información con el grupo de usuarios que lo conforman, arqueólogos, visitantes a los museos y los guías del INAH, que servirá para el modelado tridimensional con un alto grado de aproximación a la realidad de los objetos.

\section{Posibles conclusiones}

Aportaciones metodológicas: Los resultados obtenidos servirán de base para trabajos futuros en $3 \mathrm{D}$, tanto en investigación como en trabajos de desarrollo que vinculen a la Cultura Mixteca.

Aportaciones prácticas: Los modelos se realizarán en base a la experiencia que viven los usuarios de los museos y el entendimiento que tienen con los guías y arqueólogos del INAH. Dichos modelos podrán ser utilizados por investigadores, educadores, estudiantes y público en general, para tener una concepción mejor del arte generado por la cultura mixteca.

\section{Referencias}

[1] B. Schilit, N. Adams, y R. Want, 1994. Context-aware computing applications. , 1st International Workshop on Mobile Computing Systems and Applications, pp. 85-90.

[2] D. Norman and S. Draper.1986. User Centered System Design; New Perspectives on Human-Computer Interaction. L. Erlbaum Associates Inc., Hillsdale, NJ, USA.

[3] D. Norman. 2002.The Design of Everyday Things. Basic Books, Inc.

[4] M. Winter, Cerro de las minas, Oaxaca, Arqueología Mexicana.

[5] M. Winter. 1997. "La arqueología de los Valles Centrales de Oaxaca", en Arqueología mexicana, 26: 6-15, México.

[6] R. Harper, T. Rodden, Y. Rogers, and A. Sellen.2008.Being Human: Human-Computer Interaction in the year 2020. Microsoft Research Ltd.

[7] Modelados 3D, http://www.inah.gob.mx/es/boletines/6502o-concurso-nacional-del-inah-pon-a-mexico-en-el-mapa junio, 2016.

[8] Autodesk 3D Design, Engineering \& Entertainment Software, http://www.autodesk.com/ junio, 2016.

[9] blender.org - Home of the Blender project - Free and Open 3D creation software, https://www.blender.org/ mayo, 2016. 